\title{
Emergencia de infecciones por Enterococcus sp resistente a vancomicina en un hospital universitario en Chile
}

\author{
Alberto Fica C., María Irene Jemenao P., Paola Bilbao V., Gloria Ruiz R., Andrea Sakurada Z., \\ Edith Pérez de Arce O., Isabel Zúñiga B. y Macarena Gompertz G.
}

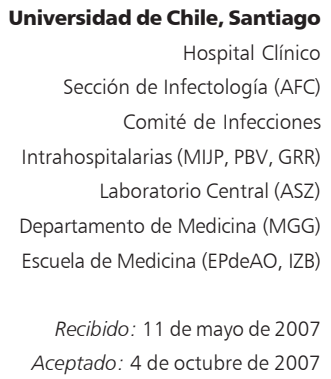

Correspondencia a: Alberto Fica Cubillos afica@redclinicauchile.cl

\section{Emergency of vancomycin-resistant Enterococcus infections in a teaching hospital in Chile}

An active surveillance of vancomycin-resistant enterococci (VRE) intestinal colonization in selected group of patients has been developed in Chile since year 2000. Nevertheless, no reports of clinical cases have been published. Aim. To describe main clinical and microbiological features of patients infected by VRE in a tertiary-level teaching Hospital. Patients and methods. Intestinal and clinical samples positive to VRE were provided by laboratory, and a retrospective analysis of potential risk factors, clinical features, treatment and outcomes was performed. Study encompassed years 2001 to 2006. Main results. 23 cases of infections were identified, all cases occurring during 2005 and 2006. Incidence rate was 0.07 and 0.09 cases per 1000 occupied bed-days, respectively. The mean age was $62.0 \pm 17$ years. A significant proportion of patients had cancer (39.1\%), recent surgical procedures $(54.1 \%)$, were on dialysis $(26.1 \%)$, or were using steroids $(26.1 \%)$. Most patients had received 2 or more antimicrobial (87\%), almost a third represented transfers from other hospitals and an additional $22 \%$ readmissions before 30 days of latest discharge. Patients were mainly hospitalized in the ICU $(60.9 \%)$ but nearly $30 \%$ were associated exclusively to nephrological or onco-hematological wards. Clinical manifestations included bacteremia (30.4\%), surgical site infections or abscesses (26.1\%), urinary tract infections $(26.1 \%)$ and others. . Three patients (13\%) did not have symptoms. After identification was possible, all isolates were identified as E. faecium ( $82.6 \%$ of total), the rest as Enterococcus sp. Most strains showed intermediate susceptibility to vancomycin $(66.7 \%)$. For 14 strains studied both with vancomycin and teicoplanin, , phenotype Van B was predominant $(85.7 \%)$, followed by VanA $(7.1 \%)$ and VanB/VanD type (7.1\%). No molecular studies were performed. Fifteen patients $(65.4 \%)$ received a surgical and/or medical treatment. A favorable response was observed in $80 \%$ of these cases. Eight patients were not treated $(34.8 \%)$, in 2 cases because of a rapidly-fatal infection. The global risk-fatality ratio for VRE infections was $13 \%$ and increased to $42.9 \%$ in patients with bacteremia. Microbiological eradication was documented in $52.2 \%$.

Key words: Emergency, vancomycin-resistant Enterococus, infection, intestinal colonization, clinical features, treatment, outcomes.

Palabras claves: Emergencia, Enterococcus resistente a vancomicina, infección, colonización intestinal, manifestaciones clínicas, tratamiento, evolución, resultados.

\section{Introducción}

$\mathrm{L}$ as infecciones por Enterococcus sp resistente a vancomicina (ERV) han aumentado progresivamente en las últimas dos décadas, aparejadas con aumento de la mortalidad, estadía hospitalaria y costos económicos ${ }^{1,2}$. Así, las infecciones por este microorganismo fueron asociadas a un exceso de mortalidad de $6 \%$, seis días de hospitalización adicional, cerca de 12 mil, $10 \%$ adicional en procedimientos quirúrgicos y $11 \%$ adicional de internación en unidades de cuidados intensivos $(\mathrm{UCI})^{2}$. Las infecciones por
ERV ocurren en el ambiente hospitalario, en pacientes debilitados o invadidos y se expresan en forma de bacteriemias, infecciones urinarias, colecciones sépticas o abscesos ${ }^{1-4}$.

En Chile, las primeras infecciones asociadas a ERV Se registraron el año 2000 y no se dispone de información adicional que de cuenta de la magnitud del problema $^{3}$. Para contener la emergencia de este problema se adoptó ese año una norma nacional de vigilancia activa de portación intestinal en pacientes de UCI y de casos clínicos (Circular 4/C28 del 9 de mayo de 2000) y directrices para el manejo de estos casos con la finali- 
dad de evitar la transmisión cruzada. Además, se hizo un fuerte énfasis en el uso racional de antimicrobianos, especialmente, sobre vancomicina ${ }^{3}$.

El objetivo de este trabajo es presentar las características de una serie de pacientes con infecciones clínicas por ERV, observadas desde el inicio de nuestro sistema de vigilancia, el año 2001.

\section{Pacientes y Métodos}

Criterios de inclusión. Se incluyeron todos aquellos pacientes internados que presentaron aislados clínicos de Enterococcus sp con susceptibilidad intermedia o resistencia a vancomicina. La lista de casos fue provista por el laboratorio de Microbiología. Se incluyeron pacientes sintomáticos $\mathrm{y}$ asintomáticos $\mathrm{y}$ se excluyeron los casos de portación intestinal. El período de estudio abarcó desde el año 2001 hasta el 2006.

Variables clínicas. Se registraron los datos biodemográficos de los pacientes, sus antecedentes mórbidos, cirugía en los últimos 12 meses, fecha de ingreso, traslado desde otro centro o reingreso al hospital, fecha de diagnóstico de la infección por ERV, datos microbiológicos, antibiograma y, en aquellos casos en que estuvo disponible, el valor de CIM para vancomicina. También se evaluó el uso de procedimientos médicos invasores o tratamientos de riesgo tales como sondas intestinales, procedimientos endoscópicos digestivos o respiratorios, ventilación mecánica, CVC, catéter vesical, nebulizaciones y un registro completo de los antimicrobianos utilizados antes del diagnóstico de la infección. Los sitios de infección fueron catalogados como: bacteriemia, neumonía, infección de herida operatoria, colección o infección urinaria. La asociación con ventilación mecánica o catéter urinario fue precisada. Cada caso clínico fue catalogado como sintomático o asintomático, como infección polimicrobiana (si en el mismo sitio de cultivo creció otro microorganismo) o monomicrobiana, y también se registró si había otro foco infeccioso clínico concomitante. Además, se buscó en los registros del Comité de Infecciones Intrahospitalarias (IIH) si el paciente tenía portación intestinal de ERV, había sido contacto de un paciente portador o si se encontraba hospitalizado en una sección en la que en el mismo mes se hubiera detectado un portador. Las unidades de hospitalización se dividieron arbitrariamente en Unidad de Pacientes Críticos (UPC) incluyendo la propia UCI y las salas intermedias, en Unidad Hemato-Oncológica (UHO) y en Sala Nefrológica (incluyendo en este rubro el Intermedio de Nefrología).
Incidencia de ERV en infecciones clínicas. Los casos con muestras clínicas positivas para ERV permitieron construir un indicador de su frecuencia expresada en incidencia/1.000 días camas ocupadas para cada año, descontando los días camas del Departamento de Psiquiatría. Las tasas obtenidas fueron contrastadas con las tasas de casos nuevos de portación intestinal de ERV, disponibles en los informes semestrales de vigilancia epidemiológica del Comité de IIH.

Detección de portadores intestinales de ERV y estudio microbiológico de muestras positivas. Desde el año 2001 se efectúa un estudio periódico de vigilancia de portación intestinal de ERV en grupos seleccionados de pacientes. El Ministerio de Salud de Chile (MINSAL) ha recomendado efectuar tal estudio a comienzos de cada mes en todos los pacientes que cumplen 5 o más días de hospitalización y que estén presentes el día del estudio en cualquiera de las unidades de cuidados críticos o intermedios del hospital. Nuestra institución incluyó además el estudio de los pacientes hospitalizados en salas de Nefrología y en la Unidad Hemato-Oncológica. Durante el período de estudio, los pacientes portadores de ERV fueron seguidos con la obtención de una muestra semanal. La vigilancia activa se efectuó, además, en otros dos grupos de pacientes: los trasladados desde otro recinto hospitalario y los contactos, definidos operativamente, como aquellos pacientes que permanecieron más de 24 horas compartiendo la misma habitación con un caso índice, antes de haberse adoptado las medidas de aislamiento. Las muestras fueron transportadas en medio Stuart al laboratorio, sembradas en agar telurito (Enterococcosel, BBC) suplementado con 6 $\mu \mathrm{g} / \mathrm{mL}$ de vancomicina (Oxoid) a $35^{\circ} \mathrm{C}$ y leídas hasta 72 horas de observación. Se identificaron las colonias de color negro (esculina positivas), las que fueron traspasadas a agar sangre cordero (Biomerieux Marcy L'Etoile, France). La identificación se efectuó en caldo sal $6,5 \%$ - bilis esculina y, desde agosto de 2006, se incorporó un sistema de microgalerías API 20 Strept (Biomerieux Marcy L'Etoile, France). La diferenciación entre E. gallinarum y E. faecium se efectuó mediante motilidad entre lámina y laminilla y movilidad en agar blando. En el caso de muestras clínicas, la identificación se efectuó en forma similar a lo descrito para las muestras de vigilancia.

Estudio de susceptibilidad. Se efectuó en forma estándar en agar Müller Hinton (BioMerieux) con un disco de $30 \mu \mathrm{g}$ de vancomicina, lectura a las 24 horas de incubación a $35^{\circ} \mathrm{C}$ y siguiendo las instrucciones del CLSI para los años 2005 y 2006 $6^{5,6}$. Se consideró como resistencia un halo de inhibición $<17 \mathrm{~mm}$ y estas cepas fueron analizadas, posteriormente, mediante E-test (AB 
Biodisk, Solna, Sweden). Se consideró como cepa susceptible aquella con una CIM $\leq 4 \mu \mathrm{g} / \mathrm{mL}$, como cepa de susceptibilidad intermedia aquellas con CIM de 6 a 24 $\mu \mathrm{g} / \mathrm{mL}$ y como cepas resistentes aquellas con CIM $\geq 32 \mu \mathrm{g} / \mathrm{mL}$. El antibiograma para ERV incluyó habitualmente ampicilina, gentamicina (disco de $120 \mu \mathrm{g}$ ), ciprofloxacina, tetraciclina y nitrofurantoína. En algunos casos se contó también con estudio de susceptibilidad ante teicoplanina y/o linezolid mediante difusión en agar. El reconocimiento fenotípico del perfil de resistencia VanA, VanB o Van D se basó en los rangos publicados de CIM para vancomicina y en la susceptibilidad o resistencia por difusión ante teicoplanina ${ }^{3}$. Se catalogó como fenotipo VanA aquel con resistencia simultánea a ambos glicopeptidos, como VanB, aquel con CIM entre $6 \mathrm{y}>256 \mu \mathrm{g} / \mathrm{mL}$ para vancomicina y susceptibilidad ante teicoplanina y como fenotipo VanD aquella condición con un valor CIM entre 64 y $128 \mu \mathrm{g} / \mathrm{mL}$ para vancomicina y susceptibilidad ante teicoplanina. No se efectuaron estudios moleculares en los aislados de ERV.

Tipos de tratamiento y evaluación de la respuesta terapéutica. Se consideró como tratamiento médico el uso aislado, o en combinación, de cualquier antimicrobiano activo según el informe del antibiograma, y como tratamiento quirúrgico el uso de drenajes o aseos quirúrgicos para tratar una colección o herida operatoria infectada. Se precisó si el paciente recibió alguna combinación sinérgica para Enterococcus sp y se consideró tal si se asoció una terapia de gentamicina con glicopeptidos, incluso vancomicina. La respuesta al tratamiento fue catalogada como favorable si se observó una mejoría clínica y de laboratorio durante cualquiera de las estrategias escogidas; como fracaso si el paciente falleció durante el tratamiento o permaneció en condiciones clínicas estacionarias, y como no evaluable si el paciente no recibió tratamiento para esta infección. La respuesta microbiológica fue catalogada como erradicación, si en el mismo sitio del cultivo original, un cultivo posterior al tratamiento demostró la ausencia de ERV o la respuesta favorable hizo presumir su erradicación. Se definió la respuesta microbiológica como persistencia en caso de que el primer cultivo posterior al término del tratamiento demostrara la presencia de ERV. Aquellos casos en los cuales no hubo un cultivo de seguimiento y no fue posible presumir su erradicación, fueron catalogados como una respuesta microbiológica no evaluable.

Evaluación de la mortalidad. Fue evaluada hasta el egreso hospitalario y también en los subgrupos tratados y no tratados.
Medidas de control. Se aplicaron varias estrategias de control para evitar la diseminación de ERV, incluyendo la vigilancia de portación intestinal ya comentada, el aislamiento en pieza exclusiva o cohortes de los casos clínicos o de portación (en la medida de lo posible), la aplicación de precauciones de contacto, incluyendo pecheras desechables y desinfección ambiental dos veces al día, instructivos para el traslado del paciente y su atención en unidades de apoyo. En nuestra institución se aplica, además, un programa de uso racional de antimicrobianos con un sistema de supervisión activa y que incluye vancomicina, carbapenémicos, sulbactam/cefoperazona y otros compues$\operatorname{tos}^{7}$.

Análisis estadístico. Se aplicó la prueba bilateral de Fisher para el análisis de potenciales factores asociados a una respuesta no favorable o fracaso al tratamiento. Las variables continuas (edad, etc) fueron transformadas en variables categóricas para este tipo de análisis. Para evaluar tendencias en las tasas de portación de ERV o de infecciones clínicas durante el período 2001-2006, se efectuó un análisis de correlación lineal no paramétrico (coeficiente de correlación de Spearman).

\section{Resultados}

Fue identificado un total de 23 infecciones con manifestaciones clínicas en el mismo número de pacientes. Cuatro casos adicionales no fueron incluidos en este estudio, por diferentes causas: dos de ellos por extravío de la ficha clínica (ninguno de ellos asociado a bacteriemias), un caso de infección urinaria baja detectada en una muestra ambulatoria poco después del alta del paciente y que no fue tratada (año 2004), y un caso de infección de tejidos blandos con información microbiológica extraviada y que fue tratado con aseos locales y buena evolución.

Los casos sintomáticos fueron registrados los años 2005 (n: 9, 39,1\%) y 2006 (n: 14, 60,9\%). No se observaron casos entre el 2001 y 2004. La incidencia el año 2005 fue de 0,07 casos/1.000 días camas ocupadas y de 0,09 en el año 2006 (Figura 1). La aparición de casos clínicos fue casi simultánea a la detección de portadores intestinales. Los primeros casos de portación aparecieron el año 2004 (cuatro casos) y los sintomáticos desde el 2005. La tasa de portación fue superior a la de casos clínicos (1,4 vs 0,09/1.000 el año 2006); el incremento de ambas tasas fue significativo a lo largo del período (coeficiente de correlación de Spearman r: 0,94 valor $p<0,01$ y $r: 0,85$ valor $p<0,05$, respectivamente). 
Características de la población estudiada. La edad promedio de los pacientes fue de 62,0 años (DS 17 años; rango 15 a 84). Co-morbilidades: cáncer hematológico o neoplasias sólidas $39,1 \%$, pacientes en diálisis $26,1 \%$, usuarios de corticoesteroides $26,1 \%$, eventos vasculares cerebrales y cirugía actual o reciente 17,4 y $56,5 \%$, respectivamente (Tabla 1 ).

Un tercio de los pacientes había sido trasladado desde otro centro hospitalario y cerca de $22 \%$ adicional representó reingresos antes de 30 días desde el alta. Con frecuencia los pacientes tenían antecedentes de uso de sonda intestinal, ventilación mecánica, catéter urinario, CVC, o procedimientos endoscópicos digestivos o respiratorios (Tabla 1).

Ochenta y siete por ciento había recibido múltiples tratamientos antimicrobianos y de amplio espectro (Tabla 1), incluyendo cefalosporinas (87\%), metronidazol o carbapenémicos $(60,9 \%)$, aminoglucósidos $(47,8 \%)$, fluconazol $(43,5 \%)$ o quinolonas $(34,8 \%)$. Sesenta por ciento había recibido vancomicina oral o ev (Tabla 1).

Un bajo número de pacientes había presentado infecciones previas por Staphylococcus aureus resistente a meticilina $(\mathrm{n}=4,17,4 \%)$ o por Clostridium difficile $(\mathrm{n}=2 ; 8,7 \%)$.

Salas de hospitalización y portación previa de $E R V$. El $60,9 \%$ de los pacientes estuvo hospitalizado en la UPC en algún momento antes de la infección (Tabla 2), 13,0\% en la Unidad Hemato-Oncológica y casi $20 \%$ en salas nefrológicas (Tabla 2). Cerca de la mitad de las infecciones observadas no tenían portación previa de ERV en los estudios de vigilancia y sólo un tercio presentaba este antecedente. En contraste, $87,0 \%$ presentaban el antecedente de haber estado en una unidad con casos conocidos de portación (Tabla 2). Durante el año 2005, 3\% (n: 4/134) de los portadores intestinales conocidos desarrolló una infección clínica y en el 2006 alcanzó a 2,3\% (n: 4/174).

Tipo de infecciones y perfil microbiológico de los aislados. Las infecciones se distribuyeron en forma equitativa entre bacteriemias (n: 7; 30,4\%), infecciones urinarias (n: $6 ; 26,1 \%)$ y colecciones o infecciones de herida operatoria (n: $6 ; 26,1 \%$ ). Además, se observó un caso de peritonitis secundaria a perforación de colon y otro caso de colonización en una punta de CVC, sin bacteriemia asociada o síntomas atribuibles (Tabla 3). Las bacteriemias estuvieron asociadas a pacientes con patología abdominal, renal, cardio-quirúrgica o leucemia (Tabla 3). Por su parte, las infecciones urinarias se asociaron a uso de catéter urinario, en la mayor parte de los casos. Los aislados provenientes de colecciones o infecciones de herida operatoria se asociaron a patologías infra-diafragmáticas, de diversa índole, en cuatro de los seis casos (Tabla 3).

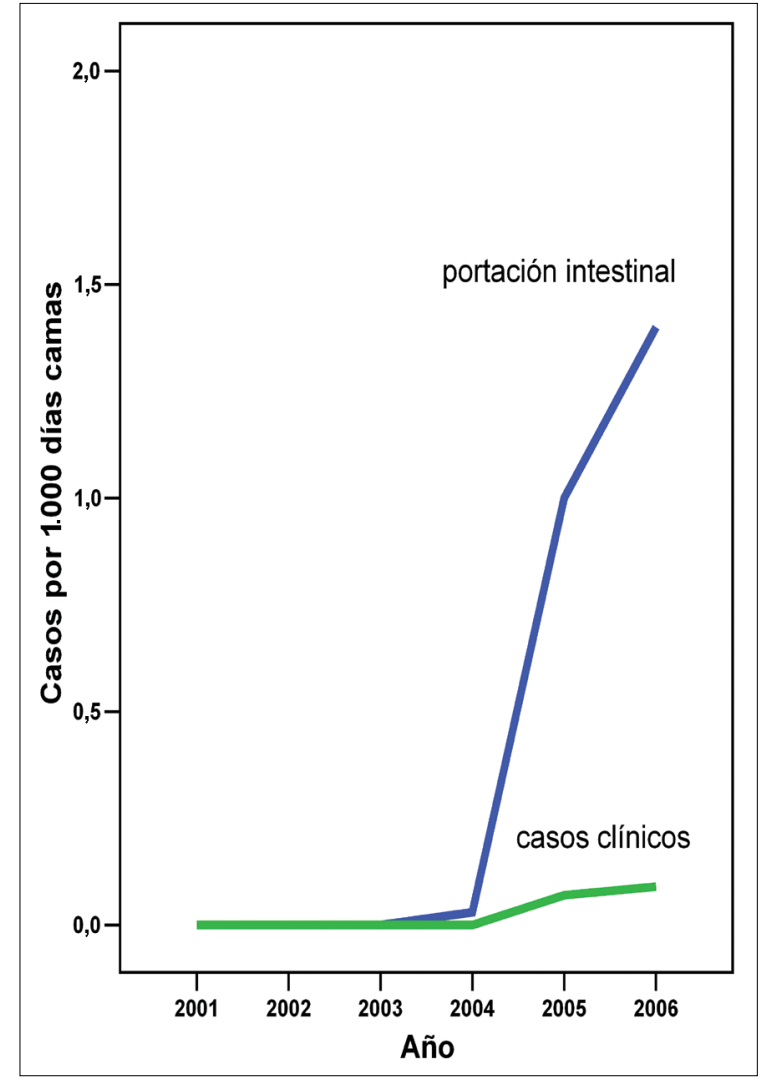

Las tres infecciones por ERV observadas en los pacientes hospitalizados en la Unidad Hemato-Oncológica se manifestaron como bacteriemias. En contraste, sólo cuatro de 20 pacientes internados en otros servicios o unidades tuvieron tal expresión (p: 0,02 por prueba bilateral de Fisher).

En nueve casos $(39,1 \%)$ existía otro foco infeccioso concomitante al asociado a ERV y en cinco de estos pacientes había un foco pulmonar (neumonía, asociada o no asociada a VM). Los otros cuatro estuvieron asociados a bacteriemia por $S$. aureus resistente a meticilina (n: 1), foco biliar (n: 1), foco intra-abdominal (n: 1) y peritonitis (n: 1); en este último caso- una paciente con leucemia- el mismo microorganismo fue identificado en diferentes focos (sangre y peritoneo).

En siete de los 23 pacientes (30,4\%), ERV fue identificado junto a otros microorganismos (cultivo polimicrobiano). Cinco de estos casos correspondieron a colecciones, abscesos o infecciones de herida operatoria y los otros dos, a una bacteriemia y una neumonía, respectivamente. El espectro de microorganismos incluyó bacilos gramnegativos entéricos o no fermentadores, Staphylococcus coagulasa negativa, S. aureus y Candida sp.
Figura 1. Tasas de portación intestinal de Enterococcus sp resistente a vancomicina y de casos clínicos/1.000 días camas ocupadas, 2001-2006. Hospital Clínico Universidad de Chile. 
Tabla 1. Características generales de los pacientes con infecciones por Enterococcus sp resistente a vancomicina

\begin{tabular}{|c|c|c|}
\hline Condición & Frecuencia & $\%$ \\
\hline Sexo $n(M / F)$ & $13 / 10$ & $56,5 / 43,5$ \\
\hline \multicolumn{3}{|l|}{ Morbilidad } \\
\hline Diabetes mellitus & 8 & 34,8 \\
\hline Diálisis & 6 & 26,1 \\
\hline Cáncer no hematológico & 5 & 21,7 \\
\hline Leucemia o linfoma & 4 & 17,4 \\
\hline Trasplante & 1 * & 4,3 \\
\hline Usuario de corticoesteroides & 6 & 26,1 \\
\hline Cirugía actual o $<12$ meses & 13 & 56,5 \\
\hline Accidente vascular encefálico actual o $<12$ meses & 4 & 17,4 \\
\hline \multicolumn{3}{|l|}{ Factores asociados a la atención } \\
\hline Traslado desde otro centro & 8 & 34,8 \\
\hline Reingreso $<30$ días desde alta previa & 5 & 21,7 \\
\hline Sonda intestinal o gástrica & 16 & 69,6 \\
\hline Cirugía abdominal & 7 & 30,4 \\
\hline Cirugía urológica o ginecológica & 1 & 4,3 \\
\hline Paciente neuro-quirúrgico & 2 & 8,7 \\
\hline Ventilación mecánica & 13 & 56,5 \\
\hline Endoscopia digestiva alta & 7 & 30,4 \\
\hline Catéter venoso central & 18 & 78,3 \\
\hline Catéter urinario & 17 & 73,9 \\
\hline Fibro-broncoscopia & 6 & 26,1 \\
\hline \multicolumn{3}{|l|}{ Uso previo de antimicrobianos } \\
\hline Cefalosporinas & 20 & 87,0 \\
\hline Metronidazol & 14 & 60,9 \\
\hline Carbapenémicos & 14 & 60,9 \\
\hline Vancomicina oral o e.v. & 14 & 60,9 \\
\hline Quinolonas & 8 & 34,8 \\
\hline Fluconazol & 10 & 43,5 \\
\hline Aminoglucósidos & 11 & 47,8 \\
\hline Uso previo de dos o más antimicrobianos & 20 & 87,0 \\
\hline
\end{tabular}

\begin{tabular}{|lcc|}
\hline \multicolumn{3}{|c|}{$\begin{array}{c}\text { Tabla 2. Salas de hospitalización ysituación de portación para } \\
\text { Enterococcus sp resistente a vancomicina }\end{array}$} \\
\hline Condición & Frecuencia & $\%$ \\
\hline Estadía en UPC & 14 & 60,9 \\
\hline Estadía en Unidad Hemato-Oncológica & 3 & 13,0 \\
\hline Estadía en Nefrología O Intermedio Nefrología & 4 & 17,4 \\
\hline $\begin{array}{l}\text { Portación previa de ERV } \\
\text { Sí }\end{array}$ & 8 & 34,8 \\
No & 11 & 47,8 \\
No estudiado & 4 & 17,4 \\
\hline Contacto con caso ERV (+) & 4 & 17,4 \\
\hline Estadía en sala con casos de portación (+) & 20 & 87,0 \\
\hline UPC: unidad de pacientes críticos. ERV: Enterococcus sp resistente a vancomicina & \\
\hline
\end{tabular}

En tres pacientes no hubo síntomas presentes o atribuibles a la infección por ERV (13\%). Dos de estos casos incluyeron pacientes con infección urinaria, uno de ellos asociada a catéter urinario; el tercero correspondió a una colonización de $\mathrm{CVC}$, sin síntomas atribuibles (Tabla 3). En otro caso, es probable que los síntomas fueran atribuibles a una bacteriemia concomitante causada por $S$. aureus.

Las infecciones se presentaron con frecuencia similar durante la primera semana desde el ingreso (n: 6/23; $26,1 \%$ ), segunda y cuarta semana (n: $9 / 23 ; 39,1 \%$ ) y después del mes de internación (n: 8/23; 34,8\%). Cuatro de los seis casos que presentaron infección en la primera semana de hospitalización correspondían a reingresos o traslados, proporción que no fue significativamente diferente a la de reingresos o traslados observados en las infecciones más tardías (datos no mostrados).

Diecinueve cepas $(82,6 \%)$ fueron identificada como E. faecium y el resto como Enterococcus sp, antes de contar con galerías de identificación.

Los aislados disponibles presentaron un perfil multiresistente con altos porcentajes de resistencia a ampicilina, ciprofloxacina y tetraciclina. Asimismo, cerca de $60 \%$ presentaba resistencia a gentamicina y $54,5 \%$ presentó simultáneamente resistencia a gentamicina y ampicilina (Tabla 4).

La CIM para vancomicina varió entre 6 y $>256 \mu \mathrm{g} / \mathrm{mL}$ en los 18 casos clínicos en los cuales se pudo contar con esta determinación. La mayor parte de las cepas presentó valores intermedios de susceptibilidad (n: 12; $66,7 \%$ ). Las restantes demostraron resistencia (n: 6; $33,3 \%$ ). Las cepas resistentes demostraron CIM de 32 $\mu \mathrm{g} / \mathrm{mL}$ en tres casos, $128 \mu \mathrm{g} / \mathrm{mL}$ en dos casos y $>256$ $\mu \mathrm{g} / \mathrm{mL}$ en los tres casos restantes.

En 19 de los 23 casos clínicos se efectuó estudio de susceptibilidad para teicoplanina, demostrándose resistencia en dos de ellos (10,5\%); una de las dos cepas tenía una CIM para vancomicina $>256 \mu \mathrm{g} / \mathrm{mL}$. En las 14 cepas que tenían simultáneamente CIM para vancomicina y evaluación de susceptibilidad a teicoplanina por difusión en agar, la frecuencia del fenotipo VanA fue de $7,1 \%$ (n: 1 ) y la frecuencia del fenotipo vanB fue de $85,7 \%$ (n: 12). Además, una cepa de E. faecium con CIM de $128 \mu \mathrm{g} / \mathrm{mL}$ y susceptible a teicoplanina pudo haber correspondido a una cepa de fenotipo VanB o VanD (Tabla 5).

Tratamiento y evolución. En dos tercios de los casos (n: $15,65,2 \%$ ) se optó por un tratamiento médico o médico-quirúrgico; en ocho casos $(53,3 \%)$ este tratamiento sólo fue con antimicrobianos, en cinco pacientes fue médico-quirúrgico $(33,3 \%)$ y en dos de casos se practicó sólo tratamiento quirúrgico. 


\section{Tabla 3. Tipo de infecciones asociadas a Enterococcus sp resistente a vancomicina}

\begin{tabular}{|c|c|c|c|}
\hline Tipo & $\mathbf{n}$ & $\%$ & Perfil del caso \\
\hline Bacteriemia & 7 & 30,4 & $\begin{array}{l}\text { Perforación intestinal ( } \mathrm{n}: 2 \text { ) } \\
\text { LED y falla renal ( } \mathrm{n}: 1) \\
\text { Paciente cardio-quirúrgico ( } \mathrm{n}: 1) \\
\text { Leucemia linfática aguda y neutropenia ( } \mathrm{n}: 1) \\
\text { Leucemia mieloide crónica y neutropenia ( } \mathrm{n}: 1) \\
\text { Leucemia mieloide aguda (n: } 1)\end{array}$ \\
\hline Infección urinaria & 6 & 26,1 & $\begin{array}{l}\text { Asociada a catéter urinario ( } \mathrm{n}: 4) \\
\text { No asociada a catéter urinario (n: 2) } \\
\text { Asintomáticos ( } \mathrm{n}: 2)\end{array}$ \\
\hline Colección o infección de herida operatoria & 6 & 26,1 & $\begin{array}{l}\text { Infección de herida abdominal en cirugía contaminada (n: 2) } \\
\text { Absceso hepático tardío en paciente operado de cáncer gástrico ( } n \text { : 1) } \\
\text { Colección intra-abdominal en paciente urológico ( } \mathrm{n} \text { : 1) } \\
\text { Colección mediastínica en paciente operado de cáncer de esófago (n: 1) } \\
\text { Absceso cerebral en paciente neuro-quirúrgico (n: 1) }\end{array}$ \\
\hline Neumonía asociada a VM & 2 & 8,7 & $\begin{array}{l}\text { Paciente neuro-quirúrgico }(n=1)>10^{6} \mathrm{ufc} / \mathrm{mL} \text { en CCAET } \\
\text { Paciente con colangitis }(n=1) 10^{5} \mathrm{ufc} / \mathrm{mL} \text { en CCAET }\end{array}$ \\
\hline Otras & 2 & 8,7 & $\begin{array}{l}\text { Peritonitis secundaria a perforación de colon en paciente en peritoneo-diálisis } \\
\text { crónica }(n=1) \\
\text { Colonización de CVC sin bacteriemia y sin síntomas atribuibles }(n=1)\end{array}$ \\
\hline Total & 23 & 100 & \\
\hline
\end{tabular}

Los 13 pacientes con tratamiento antimicrobiano recibieron: teicoplanina cinco casos $(38,5 \%)$, ampicilina, gentamicina y linezolid (dos casos con cada fármaco), nitrofurantoina (en un caso) y terapia secuencial con teicoplanina-linezolid en el caso restante.

Sólo en dos casos se aplicó una terapia sinérgica con gentamicina y glicopéptidos, incluyendo en uno de ellos la combinación con vancomicina frente a un aislado con resistencia intermedia (CIM a vancomicina: $16 \mu \mathrm{g} / \mathrm{mL})$.

Se inició el tratamiento antimicrobiano en las primeras 72 horas, desde la fecha del cultivo, en seis de los 13 pacientes tratados $(46,7 \%)$, entre cuatro y siete días en el mismo número de pacientes $(46,7 \%)$ y después de los siete días en el caso restante (a los 16 días, 7,7\%).

Ocho pacientes $(34,8 \%)$ no fueron tratados y no fueron evaluables en su respuesta terapéutica. Diversas razones estuvieron involucradas en este grupo: en dos casos una evolución grave, falleciendo el paciente antes de disponerse del informe de laboratorio, en cuatro casos estuvo involucrada una infección urinaria asintomática o de difícil interpretación sobre su relevancia clínica, en los dos casos restantes, la información del laboratorio se recibió cuando ya se había decidido suspender las medidas terapéuticas.

Se observó una respuesta favorable en 12 de los 15 pacientes que recibieron tratamiento médico o quirúr-

Tabla 4. Resistencia ante diferentes compuestos antimicrobianos en aislados clínicos de Enterococcus sp resistente a vancomicina

Compuesto (cepas estudiadas)

Ampicilina (23)

Gentamicina (22)

Ciprofloxacina (22)

Tetraciclina (22)

Ampicilina y gentamicina (22)

Tabla 5. Susceptibilidad ante glicopeptidos y fenotipo de resistencia

\section{Parámetro}

Vancomicina

Susceptibilidad intermedia

Resistencia

Total

Teicoplanina

Susceptibles

Resistentes

Total

Fenotipo

Fenotipo VanA

Fenotipo VanB

Fenotipo VanB/VanD

Total

\section{$\%$ de resistencia}

87,0

59,1

95,5

81,8

54,5 
gico $(80 \%)$. La tasa de respuesta en los siete pacientes con tratamiento médico-quirúrgico fue de $71,4 \%$ y en los ocho pacientes con tratamiento antimicrobiano exclusivo, de $87,5 \%$ (diferencia no significativa). De la misma manera, la respuesta favorable en el grupo que recibió tratamiento con teicoplanina fue de $85,7 \%$, no estadísticamente diferente a la observada en el grupo que recibió otros esquemas (75\%). El bajo número de pacientes impidió explorar estadísticamente el impacto de un tratamiento de inicio tardío $>72$ horas desde la toma del cultivo, pero todos los tratamientos iniciados sobre esta ventana de tiempo tuvieron una respuesta favorable.

Tres de los 15 pacientes tratados $(20 \%)$ se consideraron como fracasos terapéuticos; dos pacientes tuvieron nuevas complicaciones intra-abdominales de su patología de base que evolucionaron sin mejoría y la tercera fue una paciente con leucemia que falleció de una bacteriemia durante su tratamiento. El bajo porcentaje y número de pacientes tratados, sin una respuesta favorable, impidió hacer un análisis de potenciales factores asociados a este resultado.

La respuesta microbiológica fue catalogada como erradicación en 12 casos $(52,2 \%)$ y como no evaluable en el resto.

Hasta marzo de 2007, un paciente de la serie seguía hospitalizado. La mortalidad cruda hasta el egreso en los 22 pacientes restantes fue de $31,8 \%$ (n: 7 ), en mayor detalle: $21,4 \%$ (3 de 14 egresados) en los tratados $v s$ $50,0 \%$ (4 de 8 pacientes egresados) en el subgrupo no tratado; esta diferencia no fue significativa. La letalidad de la infección por ERV (tratados o no tratados) fue $13,0 \%(\mathrm{n}: 3)$ y en todas ellas estuvo asociada a bacteriemia; la letalidad específica por bacteriemia fue de $42,9 \%$ (3 de 7 casos). Una de estas muertes por bacteriemia ocurrió en el grupo tratado, a los 9 días de la infección, y las otras dos en el grupo que no recibió tratamiento. La baja frecuencia de casos impidió analizar si la letalidad estuvo asociada a un tipo específico de infección.

\section{Discusión}

Los resultados de este trabajo demuestran la rápida emergencia de las infecciones por aislados de ERV en un hospital universitario. En Chile, gracias a estudios de vigilancia liderados por la Sociedad Chilena de Infectología, se pudo identificar los primeros casos de portación intestinal de ERV hacia fines de los años 90, detectándose la existencia de cepas con susceptibilidad intermedia ${ }^{3}$. El primer reporte publicado en Chile sobre un aislado de ERV data del año 2000, correspondiendo a un caso de portación intestinal ${ }^{8}$. Posterior- mente, otros dos casos de colonización fueron reportados por Mella y cols ${ }^{9}$. Hasta ahora, no existe información oficial sobre la frecuencia de aislados clínicos de ERV y esta publicación corresponde a la primera serie de casos clínicos notificada hasta ahora. Algunos estudios efectuados en el norte del país con cepas de Enterococcus sp de diferentes muestras clínicas, recolectadas hacia el 2002, no señalan detección de aislados resistentes a vancomicina ${ }^{10}$. Sin embargo, este mismo grupo logró demostrar la circulación de aislados de Enterococcus sp, con resistencia intermedia a vancomicina, en aguas servidas de la ciudad de Antofagasta ${ }^{11}$.

En nuestra serie, la aparición de casos clínicos el año 2005 fue precedida por portación intestinal que emergiera pocos meses antes, en el año 2004. La baja sensibilidad reportada para el tamizaje intestinal, que no supera el $60 \%$, y el hecho de que sólo la mitad de los casos clínicos en nuestra serie eran portadores conocidos de ERV, demuestran la limitación de esta estrategia para controlar la aparición de casos clínicos ${ }^{12}$. La baja asociación encontrada entre portación e infección clínica puede ser explicada por la baja sensibilidad de la técnica de detección o por una baja frecuencia de muestreo. Actualmente, se exige un sólo estudio mensual de portación rectal de ERV para todos aquellos pacientes hospitalizados en unidades de cuidados intensivos por más de cinco días.

A diferencia de lo indicado por el MINSAL, nuestro hospital incluye también en la vigilancia a pacientes nefrológicos y onco-hematológicos. La importancia de estos grupos queda evidenciada por la frecuencia y/o gravedad de las infecciones por ERV en ellos. Cerca de un tercio de los casos no estuvo hospitalizado en la Unidad de Cuidados Intensivos. Más aún, los pacientes onco-hematológicos, no contemplados en el esquema actual de vigilancia, ejercieron un gran peso en las bacteriemias identificadas, y además, en su letalidad. Tres de los 7 casos de bacteriemias se asociaron a esta unidad y dos de ellos fallecieron. La estrecha asociación entre bacteriemias graves o letales y pacientes onco-hematológicos es un hecho reconocido en la literatura, con una letalidad atribuible de $37 \%$ y el desarrollo de sepsis grave o shock séptico en los días siguientes a la aparición de la bacteriemia ${ }^{13}$. Claramente, estos datos indican que las infecciones por ERV sobrepasan los límites de una unidad de pacientes críticos $\mathrm{y}$, en este sentido, la estrategia actual de vigilancia de portación subestima el riesgo nosocomial.

El perfil de los pacientes es concordante con lo publicado previamente y refuerza la estrecha asociación entre hospederos debilitados, invadidos y médicamente complejos, con este tipo de infecciones ${ }^{2,4,14,15}$. 
Además, en estos pacientes se ha ejercido una gran presión selectiva al prescribirles antimicrobianos de amplio espectro. No todos los pacientes recibieron cursos previos de vancomicina, hecho que revela la importancia adicional de la transferencia horizontal de ERV. Revisiones sistemáticas no han podido demostrar una clara asociación entre el uso de vancomicina y la adquisición de ERV ${ }^{16}$.

Las principales manifestaciones clínicas de las infecciones por ERV en esta serie correspondieron a bacteriemias, colecciones o abscesos e infecciones urinarias $^{2}$. No se observó asociación entre bacteriemia y uso de CVC, como en otras series ${ }^{15}$. A pesar de la estrecha asociación entre el género Enterococcus y el aparato gastrointestinal, urológico o ginecológico, algunas infecciones no tuvieron esta relación anatómica, destacando en nuestra serie la existencia de abscesos cerebrales, colecciones mediastínicas y neumonía asociada a ventilación mecánica.

El rol patogénico de ERV fue difícil de asignar en algunos pacientes con urocultivo positivo; en dos casos había bacteriuria asintomática. En la práctica clínica diaria es difícil, no obstante, omitir del tratamiento antimicrobiano el foco urinario cuando hay varios focos presentes.

A pesar de que no pudo determinarse la especie en todos los aislados de este trabajo, una vez que se incorporó esta posibilidad, todos resultaron ser $E$. faecium.

El tratamiento de las infecciones por ERV es diverso y depende de la localización y naturaleza de la infección. La primera fase del tratamiento es el drenaje de colecciones o abscesos, el aseo de heridas y la remoción de cuerpos extraños que pueden servir de nido a la infección. La remoción de un cuerpo extraño es importante en las infecciones asociadas a un CVC, catéter urinario o prótesis articular o valvular. En nuestra serie se detectó un caso de colonización del CVC.

Enterococcus sp presenta una resistencia intrínseca a diferentes antimicrobianos, como cefalosporinas, clindamicina y cotrimoxazol ${ }^{3}$. También presenta una resistencia adquirida a glicopéptidos, especialmente E. faecium, clasificada en varios fenotipos; dos de ellos, VanA y VanB predominan en la práctica clíni$\mathrm{ca}^{3,17}$. El fenotipo VanA se caracteriza por resistencia simultánea a vancomicina y teicoplanina y el fenotipo VanB por resistencia sólo a vancomicina.

La asociación entre resistencia a vancomicina y la especie $E$. faecium ha sido mencionada en numerosos trabajos ${ }^{4,17,18}$. La baja frecuencia del fenotipo VanA en esta serie puede ser sólo un fenómeno inicial ya que en situaciones epidemiológicas establecidas la frecuencia de VanA sobrepasa a la de VanB ${ }^{4,18}$.

En la mayor parte de los casos será necesario un tratamiento antimicrobiano. Estas alternativas incluyen cloranfenicol, linezolid, teicoplanina, nitrofurantoína, tigeciclina, daptomicina, quinupristin/dalfopristin y dalvabancina o teravancina. Algunos de ellos no son opciones en infecciones sistémicas (v. gr. nitrofurantoína), otras están limitadas por el alto porcentaje de resistencia (v.gr. tetraciclina y ciprofloxacina) o por su espectro limitado hacia cepas del fenotipo VanB (teicoplanina y dalbavancina). Asimismo, pocas de estas opciones han sido validadas en experiencias comparativas o con series de casos mayores y algunas sólo incluyen, por ahora, estudios de actividad in vitro. Los dos compuestos con mayor respaldo clínico son linezolid y quinupristin/dalfopristin, este último no disponible en Chile ${ }^{19,20}$. Teicoplanina es un compuesto bacteriostático o bactericida, según la cepa involucrada, y ha sido utilizada históricamente en infecciones graves o localizadas por Enterococcus sp, con resultados similares a otras alternativas ${ }^{21,22}$. En una serie de 63 pacientes afectados por diferentes tipos de infección por Enterococcus sp, la tasa de curación clínica con teicoplanina fue de $84 \%$; en endocarditis fue de $83 \%$ y en el subgrupo con septicemia sin endocarditis, de $64 \%$, datos similares a los de nuestra serie $^{23}$.

En nuestros pacientes, el predominio del fenotipo VanB y la ausencia de resistencia universal a ampicilina y gentamicina explican la selección frecuente de teicoplanina y de los dos compuestos tradicionales como herramientas terapéuticas. Esto traduce que aún existe un espacio pare el uso de antibacterianos convencionales. La posibilidad de una combinación sinérgica, de vital importancia en infecciones graves por la tolerancia antimicrobiana en estas especies, es remota y sólo dos veces pudo ser aplicada en nuestros pacientes.

Considerando la diversidad de pacientes involucrados, muchos de ellos graves, la respuesta global ante el tratamiento médico o médico-quirúrgico de la infección por ERV fue favorable, en un alto porcentaje de pacientes. No obstante, se observaron infecciones letales en esta serie; nuestros datos sugieren que ellas están ligadas a cuadros de bacteriemias en pacientes neutropénicos y a la oportunidad de tratamiento. Las bacteriemias por ERV se han asociado, especialmente, a pacientes neutropénicos $\mathrm{u}$ onco-hematológicos, con insuficiencia renal en diálisis, con SIDA o con falla respiratoria, y luego de una prolongada hospitalización e intensa presión selectiva efectuada por antibacterianos. Se asocian, además, a una elevada letalidad ${ }^{13}$. Sin embargo, las infecciones letales por ERV no sólo se han asociado a bacteriemias, sino también a infecciones urinarias, infecciones intra-abdominales y heridas ${ }^{2}$. 
Esta serie de pacientes revela la rápida emergencia de las infecciones por ERV en nuestro país, su diversidad clínica y asociación con una amplia gama de pacientes, no sólo con los internados en UCI. Además, sólo una fracción de ellas es precedida por un estado conocido de portación. Aunque la respuesta al tratamiento es favorable en la mayor parte de los casos, casos letales ocurren en forma perceptible. Por ahora, el fenotipo VanB predomina y ello posibilita el tratamiento con teicoplanina en algunos casos. Asimismo, algunos aislados presentan susceptibilidad a gentamicina y ampicilina, lo que permite su aplicación terapéutica, aunque, lamentablemente, las posibilidades de una combinación sinérgica son escasas.

\section{Resumen}

Introducción. En Chile, se desarrolla una vigilancia activa de portación intestinal de Enterococcus resistente a vancomicina (ERV) desde el año 2000. Sin embargo, no hay publicaciones sobre casos clínicos. Objetivo: Describir casos de infección por ERV en un hospital de nivel terciario. Pacientes y Método: Se obtuvieron de los registros del laboratorio las muestras clínicas o intestinales positivas para ERV (2001 al 2006) y se analizaron en los pacientes afectados los factores de riesgo potenciales, manifestaciones clínicas, tratamiento y evolución. Resultados: Se identificaron 23 casos (tasa de incidencia año 2005 de 0,07 y año 2006 de 0,09/1.000 días camas ocupadas). El promedio de edad fue $62,0 \pm 17$ años. Antecedentes: cáncer $(39,1 \%)$, procedimientos quirúrgicos recientes $(54,1 \%)$, hemodiálisis $(26,1 \%)$, corticoterapia $(26,1 \%)$. El $87 \%$ había recibido dos o más antimicrobianos, casi un tercio fue transferido desde otros hospitales y $22 \%$ había reingresado antes de 30 días. Los pacientes habían estado principalmente en UCI $(60,9 \%)$, el resto en salas nefrológicas $\mathrm{u}$ onco-hematológicas. Los cuadros clínicos incluyeron bacteriemias $(30,4 \%)$, infecciones del sitio quirúrgico o abscesos $(26,1 \%)$, infecciones urinarias $(26,1 \%) \mathrm{u}$ otros. Tres pacientes fueron asintomáticos (13\%). Los aislados fueron identificados como E. faecium en $82,6 \%$ del total, el resto como Enterococcus sp. El $66,7 \%$ de las cepas mostró susceptibilidad intermedia a vancomicina. En 14 cepas con estudio completo para vancomicina y teicoplanina, predominó el fenotipo $\operatorname{VanB}(85,7 \%)$, seguido de los fenotipos $\operatorname{VanA}(7,1 \%)$ y $\operatorname{VanB} / \operatorname{VanD}(7,1 \%)$. Quince pacientes fueron tratados en forma médica o médicoquirúrgica, hubo respuesta favorable en $80 \%$ de ellos. Ocho pacientes no recibieron tratamiento $(34,8 \%)$, en dos casos por una evolución fulminante. La letalidad global fue de $13 \%$ y de $42,9 \%$ en el subgrupo con bacteriemia. La erradicación microbiológica se documentó en 52,2\% y en el resto no fue evaluable. Conclusiones: Las infecciones por ERV han emergido progresiva y transversalmente en nuestro hospital, se asocian mayoritariamente al fenotipo VanB y algunas bacteriemias pueden ser letales.

\section{Referencias}

1.- Lodise T P, McKinnon P S, Tam V H, Rybak M J. Clinical outcomes for patients with bacteriemia caused by vancomycinresistant Enterococcus in a level 1 Trauma Center. Clin Infect Dis 2002; 34: 922-9.

2.- Carmeli Y, Eliopoulos G, Mozaffari E, Samore M. Health and economic outcomes of vancomycin-resistant Enterococci. Arch Intern Med 2002; 162: 2223-8.

3.- Juliet C. Estudio de susceptibilidad in vitro de Enterococcus spp. Rev Chil Infect 2002; 19 (Supl 2): S111-115

4.- Chavers L S, Moser S A, Benjamín W H Jr, Banks S E, Steinbauer J R, et al.

Vancomycin-resistant enterococci: 15 years and counting. J Hosp Infect 2003; 53: 159-71.

5.- Clinical Laboratory Standard Institute. Performance Standards for Antimicrobial Susceptibility Testing. Fifteenth informational supplement. M100-S15. Vol. $25 \mathrm{~N}^{\mathrm{o}} 1$.
6.- Clinical Laboratory Standard Institute. Performance Standards for Antimicrobial Susceptibility Testing; Sixteenth informational supplement. M100-S16. Vol $26 \mathrm{~N}^{\circ} 3$.

7.- Fica A, Luppi M, Olivares R, Brito L, Zilleruelo I, Muñoz L. Cumplimiento sobre las recomendaciones de uso y evaluación del impacto económico de un programa de uso restringido de imipenem-cilastatina. Rev Chil Infect 2006; 23: 307-15.

8.- Marovac J, Campos M I. Enterococcus faecium resistente a vancomicina. Rev Méd Chile 2000; 128: 685-6.

9.- Mella S, Sepúlveda M, Acosta P, Bello H, Domínguez M, González G, et al. Aislamiento de Enterococcus faecium resistente a vancomicina en el Hospital Clínico Regional de Concepción. Rev Chil Infect 2002; 19 : 32-6.

10.- Silva J, Asserella L, Bolados N, Herrera N, Leyton J. Resistencia a antimicrobianos en cepas de Enterococcus sp aisladas en hospitales del norte de Chile. Rev Chil Infect
2006; 23: 226-31.

11.- Silva J, Loyola P, Galleguillos J, Rodríguez Y, Colque-Navarro P, Mollby R, et al. Prevalencia de enterococos resistentes a antibióticos en aguas servidas en el Norte de Chile. Rev Méd Chile 2005; 133 : 1201-10.

12.- D'Agata E M C, Gautam S, Green W K, Tang Y W. High rate of false-negative results of the rectal swab culture method in detection of gastrointestinal colonization with vancomycin-resistant Enterococci. Clin Infect Dis 2002; 34: 167-72.

13. - Montecalvo M A, Shay D K, Patel P, Tacsa L, Maloney S A, Jarvis W R, Wormser G P. Bloodstream infections with vancomycin-resistant enterococci. Arch Intern Med 1996; 156: 1458-62.

14.- Bonten M J M, Willems R, Weinstein R A. Vancomycin-resistant enterococci: why are they here, and where do they come from? Lancet Infect Dis 2001; 1: 314-25.

15.- Peset V, Tallón P, Sola C, Sánchez E, Sarrión A, Pérez-Bellés C, et al. 
Epidemiological, microbiological, clinical, and prognostic factors of bacteremia caused by high-level vancomycin-resistant Enterococcus species. Eur J Clin Microbiol Infect Dis 2000; 19: 742-9.

16. - Carmeli Y, Samore M H, Huskins C. The association between antecedent vancomycin treatment and hospital-acquired vancomycin-resistant Enterococci. A meta-analysis. Arch Intern Med 1999; 159: 2461-8.

17.- Rice L B, Bonomo R A. Genetic and biochemical mechanisms of bacterial resistance to antimicrobial agents. En Lorian V. Ed. Antibiotics in Laboratory Medicine. $4^{\text {th }}$ Ed. Williams \& Wilkins, Baltimore,
1996. p 453-501.

18.- Bhavnani S M, Drake J A, Forrest A, Deinhart J A, Jones R N, Biedenbach D J, et al. A nationwide, multicenter, casecontrol study comparing risk factors, treatment, and outcome for vancomycinresistant and -susceptible enterococcal bacteremia. Diagn Microb Infect Dis 2000; 36: $145-58$.

19.- Moellering R C, Linden P K, Reinhardt J, Blumberg E A, Bompart F, Talbot G H. The efficacy and safety of quinupristin/ dalfopristin for the treatment of infections caused by vancomycin-resistant

Enterococcus faecium. Synercid EmergencyUse Study Group. J Antimicrob Chemother 1999; 44: 251-61.
20.- Moellering R C. Linezolid: The first oxazolidinone antimicrobial. Ann Intern Med 2003; 138: 135-42.

21.- Hayden M K, Koening G I, Trenholme G M. Bactericidal activities of antibiotics against vancomycin-resistant Enterococcus faecium blood isolates and synergistic activities of combinations. Antimicrob Agents Chemother 1994; 38: 1225-9.

22.- Martino P, Venditti M, Micozzi A, Brandimarte C, Gentile G, Santini C, et al. Teicoplanin in the treatment of Grampositive-bacterial endocarditis. Antimicrob Agents Chemother 1989; 33: 1329-34.

23.- Schmit J L. Efficacy of teicoplanin for enterococcal infections: 63 cases and review. Clin Infect Dis 1992; 15: 302-6. 